tant amplitude. A feedback circuit (a phase locked loop) adjusts frequency and phase of this oscillator to track that of the received photodetector signal. This involves some inertia to overcome intervals of inadequate received signal, but a continuous output is available in terms of the Doppler frequency and analog voltage.

\section{Very Few Photons: the Digital Cor-} relator

The Digital Correlator works well even with very low received light levels, fairly accurate velocity estimates being obtained from as little as 50 detected photons. A further advantage is its "democratic" operating principle, i.e. all received photons are treated alike. Hence statistical and information analysis may be applied to obtain the limiting accuracy of results. By comparison, threshold devices which are used in the counter and tracker introduce a degree of arbitrariness, e. g. in the level in threshold devices, which makes a similar assessment of results difficult.

Photomultiplier pulses, each one representing a single detected photon, are converted to pulses of standard amplitude and duration, and the number registered in equal time slots is recorded. Using a shift register delay line, many multipliers and stores, the digital, discrete, truncated form of the intensity autocorrelation function is accumulated in the stores. If $/(t)$ is light intensity, the function so obtained is, except for unessential factors, an estimate of the autocorrelation function:

$$
G(\tau)=\lim _{T \rightarrow \infty} \frac{1}{T} \int_{0}^{T} /(t) /(t+\tau) d t .
$$

By the Wiener-Khintchine theorem, the Fourier transformed of $G(\tau)$ is the spectrum. In the present case, the spectrum contains a maximum at the most prominent Doppler frequency $\omega_{D}$. In addition, there is instrumental broadening due to the finite width of laser beams and possibly further broadening due to velocity fluctuations, i.e. turbulence.

A trade-off is possible between measurement time and accuracy. For best accuracy, one uses a long accumulation time, perhaps many seconds, to overcome the effects of digitalization and genuine noise. On the other hand, fair estimates of velocity may be obtained after short accumulation, e. g. over a fraction of a microsecond. A number of algorithms exist to extract velocity information out of correlograms in various circumstances. This is not restricted to average velocity but includes higher moments of the velocity distribution as well.

\section{State of the Art}

Laser Doppler anemometry offers a nonintrusive method of measuring fluid velocities, suitable for hostile environments where material probes cannot endure, e. $\mathrm{g}$. in flames, explosions, combustion, acids. Besides the optical systems discussed reference beam, dual focus, cross-beam there are several others, including telescopic with up to $1 \mathrm{~km}$ range and glass fibre systems capable of measuring inside blood vessels. Other refinements in optical systems allow the flow direction to be distinguished (referring to equation (4), positive and negative $\omega_{D}$ cannot be distinguished experimentally) and the simultaneous measurement of two or three components. Velocities measured range from $0.1 \mu \mathrm{m} / \mathrm{s}$ to $1000 \mathrm{~m} / \mathrm{s}$. Once assembled, equipment works reliably and can be operated by unskilled labour.

On the other hand, further development may still be required if one wants to measure at very large/very small dimensions, if extremely high/extremely low velocities are expected, if high accuracy (relative error smaller than $10^{-3}$ ) or good data on the statistics of turbulence are asked for, or if flow at not easily accessible locations is to be studied. Examples are measurements in the boundary layer at the wing of airborne aircraft, near the propeller of a ship while moving on the ocean, or between blades of high-speed turbines.

Not surprisingly, laser Doppler anemometry has revitalized several areas of fluid mechanics research. Besides the topics mentioned already, it provides a means of studying two-phase flows, for example bubbles in water, where the velocities of both components may be determined separately, flames (with graphite particles), sediment transport in rivers and oceans, powdered materials in air and others. Another field is the study of turbulence which is now possible with a higher degree of sophistication. An area that has attracted the curiosity of many physicists is that of hydrodynamic instabilities ${ }^{4}$ ). In some well-defined geometries, the transition from laminar to turbulent flow occurs via several intermediate modes of fluid flow, characterized by well-defined frequencies and wave numbers. A first step towards interpreting these phenomena is a theoretical model due to Landau which is formally analogous to the mean-field theory of continuous phase transition due to Landau and Lifschitz.

\section{BIBLIOGRAPHY}

1. Durst F., Melling A. and Whitelaw J.H., Principles and Practice of Laser Doppler Anemometry (Academic Press) 1976.

2. Durrani T.S. and Greated C.A., Laser Systems in Flow Measurement (Plenum Press) 1977.

3. Cummins H.Z. and Pike E.R., (Eds.) Photon Correlation Spectroscopy and Velocimetry (Plenum Press) 1977.

4. Swinney H.L. and Gollub J.P. "The Transition to Turbulence", Physics Today 31 (1978) 8, p. 41.

\title{
A Breadth of Vision
}

Wolfgang Gentner, founder member of EPS and member of the first Executive Committee, has died at the age of 74. A keen promoter of European co-operation in physics, Gentner's influence has been felt in the development of a number of European scientific collaborations.

Basically a nuclear physicist, he was one of the first in the Federal Republic of Germany to look towards higher energies, starting the first cyclotron there of $20 \mathrm{MeV}$ at Heidelberg. He was a vigorous supporter of CERN and for five years headed the Division that brought the CERN $600 \mathrm{MeV}$ sychro-cyclotron into operation and launched the programme of physics research. He served on the CERN Scientific Policy Committee and as delegate to Council, being elected President from 1972-74. His particular interest was in highly unstable nuclei and excited states.

$\mathrm{He}$ was also interested in geology and in the isotopic concentrations of matter and the clues they give to the origin of the Universe. With his collaborators, he was the first in Europe to study moon rocks. He became an expert on dating and was active in introducing modern physical techniques
- tracer methods, thermally induced photoluminescence, track sensitive methods, etc., to the solution of archaeological puzzles. He was able to trace the origins of the silver to be found in old coins and by applying tracer techniques to identifying the origins of pottery, proved that trade in ancient times was much freer than had been supposed.

With his breadth of interests, he was an energetic promoter of the inter-disciplinary and cultural aims of the European Science Foundation. He was closely involved in the foundation of the Laue-Langevin Institute in Grenoble and equally enthusiastic over the creation of Emblab. He had special connexions with Israel and was his country's representative on the Minerva collaboration with Rehovot.

Founder of the Max-Planck-Institut für Kernphysik in 1958, he was until 1972 its Executive Director. In all his activities, he was particularly well-known for his encouragement of young physicists, open to the unconventional approach and welcoming personalities of widely different background. His breadth of vision will remain an inspiration. 